\title{
Diabetic Macular Edema: Fundus Autofluorescence and Functional Correlations
}

\author{
Stela Vujosevic, ${ }^{1}$ Margherita Casciano, ${ }^{2}$ Elisabetta Pilotto, ${ }^{2}$ Barbara Boccassini, ${ }^{1}$ \\ Monica Varano, ${ }^{1}$ and Edoardo Midena ${ }^{1,2}$
}

Purpose. Diabetic macular edema (DME) shows variable clinical characteristics with unpredictable results to local treatment, probably reflecting different phenotypes. The purpose of this study was to evaluate the role of structural and functional macular imaging in the characterization of DME patterns.

Methods. One hundred fifty-one eyes of 92 diabetic patients with untreated clinically significant macular edema (CSME) underwent best corrected visual acuity (BCVA) determination (logMAR), slit lamp biomicroscopy; fluorescein angiography; optical coherence tomography (OCT; mean central retinal thickness [CRT], volume, and DME pattern); fundus autofluorescence (FAF; absent or increased [i]FAF, single or multiple spots; iFAF area quantification); and microperimetry. Linear correlation, data agreement and three-way analysis of covariance were used for statistics.

Results. Thirty-five (23.2\%) eyes had normal FAF; and 116 eyes had iFAF: 48 (31.8\%) single-spot iFAF, 68 (45\%) multiple-spot iFAF. Retinal sensitivity in areas with iFAF was $11.5 \pm 5.3 \mathrm{~dB}$ (vs. $15.1 \pm 3.9 \mathrm{~dB}$ in normal areas, $P<0.005$ ). Retinal sensitivity of the central field was $15.1 \pm 3.9 \mathrm{~dB}$ in normal FAF, $12.4 \pm 4.8 \mathrm{~dB}$ in single-spot $\mathrm{iFAF}$ and $11.4 \pm 4.9 \mathrm{~dB}$ in multiple-spot iFAF $(P<0.05)$. OCT CRT and volume were not significantly different between the FAF groups. OCT volume correlated to OCT CRT $(r=0.68)$, retinal sensitivity in iFAF $(r=-0.50)$ and BCVA $(r=0.42)$. Cystoid OCT pattern and FA edema patterns correlated with iFAF presence $(P<0.0001)$.

Conclusions. In CSME, FAF correlates better with OCT patterns and central field microperimetry rather than with visual acuity. FAF is a rapid, noninvasive technique that may give new insight into the evaluation of DME. The validity of FAF in the follow-up and treatment outcomes in DME remain to be assessed. (Invest Ophthalmol Vis Sci. 2011;52:442-448) DOI:10.1167/iovs.105588

$\mathrm{D}$ iabetic macular edema (DME) is the major cause of vision loss in diabetic patients. ${ }^{1,2}$ Approximately $14 \%$ of all diabetics are affected by DME, and the 10-year incidence of DME in patients with newly diagnosed type 2 diabetes is approximately $20 \% .^{3,4}$ The gold standard for diagnosing DME remains fundus slit lamp biomicroscopy. ${ }^{5}$ Recently, optical coherence tomography (OCT) has been shown to be a valid method for early detection of DME, and its use has become a new morphologic gold standard in everyday clinical practice and ran-

From the ${ }^{1}$ Fondazione G. B. Bietti per l'Oftalmologia, IRCCS (Istituto Ricerca e Cura a Carattere Scientifico), Rome, Italy; and the ${ }^{2}$ Department of Ophthalmology, University of Padova, Padova, Italy.

Submitted for publication March 25, 2010; revised July 5, 2010; accepted July 30, 2010.

Disclosure: S. Vujosevic, None; M. Casciano, None; E. Pilotto, None; B. Boccassini, None; M. Varano, None; E. Midena, None

Corresponding author: Stela Vujosevic, Fondazione G.B. Bietti, IRCCS, 00198 Rome-Italy; stela.vujosevic@unipd.it. domized clinical trials. ${ }^{6-8}$ However, OCT has failed to become a surrogate for evaluation of visual function in DME patients, as it shows just moderate correlation to best corrected visual

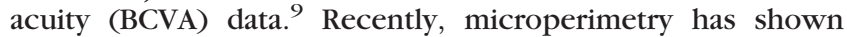
promising results in the functional evaluation of the macula, with good correlation to OCT. ${ }^{10-16}$ The role of fluorescein angiography (FA) in DME remains mostly in the assessment of capillary nonperfusion. ${ }^{17}$

Fundus autofluorescence (FAF) is a recent technique mostly used in the evaluation of age-related maculopathy and macular dystrophies. ${ }^{18-21}$ FAF is thought to derive from lipofuscin in retinal pigment epithelial (RPE) cells, reflecting some aspect of RPE function and integrity. ${ }^{20,21}$ In the macular area, the FAF signal is reduced at the fovea because of its absorption by the luteal pigment, whereas the signal is relatively increased in the parafoveal area, although still reduced when compared with the diffuse background signal in the more peripheral retinal areas. $^{20}$ FAF showed $81 \%$ sensitivity and $69 \%$ specificity in detecting cystoid macular edema, offering a rapid and noninvasive technique in the study of this disease. ${ }^{22}$ Cystoid macular edema is a common feature of different choroidal and retinal disorders. It is also a common complication of DME. FAF characteristics are still poorly known in DME. The principal purpose of this study was to evaluate FAF patterns in DME and to determine the correlation with functional parameters, such as BCVA and microperimetry. The secondary purpose was to determine the correlation of FAF patterns with FA and OCT of DME.

\section{MeTHODS}

One hundred fifty-one eyes of 92 consecutive diabetic patients with untreated clinically significant macular edema (CSME) were included in the study. All patients were recruited from Diabetic Retinopathy Clinic at the Department of Ophthalmology, University of Padova. The inclusion criteria were men or women with type 1 or 2 diabetes mellitus (DM) and previously untreated CSME, defined according to the ETDRS protocol on stereo fundus photography, and confirmed on OCT. ${ }^{23}$

The exclusion criteria were any type of previous macular treatment (macular laser photocoagulation, vitrectomy, intravitreal steroids, and/or antiangiogenic drugs); presence of subfoveal hard exudates; significant media opacities that precluded fundus examination or imaging; any intraocular surgery at least 6 months before the study involvement; and ischemic maculopathy.

A detailed written consent form (specifying the purpose of the research study and any diagnostic procedure) was obtained from all patients, as well as approval from our institutional ethics committee. The study was conducted in accordance with the tenets of the Declaration of Helsinki.

The patients underwent a complete ophthalmic examination, with determination of BCVA, anterior segment examination, indirect ophthalmoscopy, and 90-D lens biomicroscopy. Thereafter, autofluores- 
cence, FA, OCT, fundus photography, and microperimetry were performed in each patient.

\section{Study Procedures}

Visual Acuity. Distance BCVA for each eye was measured by a trained examiner using the standard Early Treatment Diabetic Retinopathy Study (ETDRS) protocol at $4 \mathrm{~m}$ distance with a modified ETDRS distance chart transilluminated with a chart illuminator (Precision Vision, La Salle, IL). ${ }^{24}$ Visual acuity was scored as the total number of letters read correctly and converted to the logarithm of the minimum angle of resolution (logMar).

Fundus Autofluorescence. FA was recorded with a confocal scanning laser ophthalmoscope (Heidelberg Retinal Angiograph 2; Heidelberg Engineering, Heidelberg, Germany), using blue wavelength (solid-pumped laser; $488 \mathrm{~nm}$ ) for excitation, whereas emitted light was detected above $500 \mathrm{~nm}$ because of a barrier filter. The optical and technical principles of the HRA have been described in detail. ${ }^{25,26}$ To amplify the autofluorescence signal of the final image, 15 acquired images were aligned, and a mean one was calculated from these, after detection and correction of eye movements were performed by image analysis software. Digital images were saved on hard disc for further analysis and processing. FAF images were graded for different foveal patterns (normal, single-spot increased, and multiple spots-increased iFAF; Fig. 1)

Decreased foveal FAF (due to the blockage caused by luteal pigment) was considered normal. In addition, the extension of the area with iFAF was manually outlined with the mouse-driven arrow on the computer screen, using the scanning laser ophthalmoscope software (Heidelberg Eye Explorer software; HRA 2; Heidelberg Engineering) All values were automatically calculated and reported in square millimeters. Grading of FAF images was performed independently by two masked readers (SV, EP), and in case of disagreement, a senior retina specialist (EM) acted as arbitrator

Stereo Fundus Photography and FA. In all patients after adequate dilation, color stereoscopic fundus photographs and FA of ETDRS field 2 were taken by a trained photographer with the same $35^{\circ}$ fundus camera (TRC 50IA; Topcon, Tokyo, Japan) and saved in JPEG format. ${ }^{23}$ Two retinal specialists independently graded, in a masked fashion, each pair of images on a 17 -in. monitor dedicated to diabetic retinopathy screening. CSME was graded according to the ETDRS protocol. ${ }^{23} \mathrm{FA}$ images of the macula were graded for capillary loss, presence and extent of fluorescein leakage, and presence of a cystoid leakage pattern. Eyes presenting with macular ischemia extending beyond the foveal avascular zone (250-300 $\mu \mathrm{m}$; ischemic maculopathy) were excluded. ${ }^{27}$

Optical Coherence Tomography. OCT scanning was performed (Stratus OCT; Carl Zeiss Meditec, Oberkochen, Germany, with the system ver. 4.1[0052] software). The scanning protocol used for this study was the Fast Macular Thickness program that creates a retinal map algorithm consisting of six radiating cross- sectional scans, each 6-mm length, that produce a circular plot in which the foveal zone is the central circular zone $1.00 \mathrm{~mm}$ in diameter. For the purpose of this study, retinal thickness in the central millimeter was used as the OCT measurement of foveal thickness (central retinal thickness). Moreover, four additional line scans, $6 \mathrm{~mm}$ in length, were performed centered on the fovea at $0^{\circ}$, $45^{\circ}, 90^{\circ}$, and $135^{\circ}$. These images were saved on hard disc for edema pattern classification, according to the classification proposed by Otani et al. ${ }^{28}$ : cystoid pattern, spongelike pattern (noncystoid), and serous neuroretinal detachment (SNRD).

Microperimetry. Microperimetry was performed on all subjects (MP1 Microperimeter; Nidek, Gamagori, Japan). This instrument is described in detail elsewhere ${ }^{29}$ For the purpose of this study, the following parameters were used: a fixation target consisting of red ring, $1^{\circ}$ in diameter; white, monochromatic background at 4 asb, stimulus size Goldman III, with 200-ms projection time; and a customized radial grid of 45 stimuli covering the central $12^{\circ}$ (centered on the fovea), $1^{\circ}$ apart for the inner stimuli and $2^{\circ}$ apart for the outer stimuli. The starting stimulus light attenuation was set at $10 \mathrm{~dB}$. A 4-2-1 double-staircase strategy was used with an automatic eye tracker that compensates for eye movements. ${ }^{29,30}$ Pretest training was performed, and 5 minutes of mesopic visual adaptation was allowed before the test began. All subjects underwent microperimetry with dilated pupils Mean retinal sensitivity was evaluated over areas with iFAF and within the central $4^{\circ}$, covering $\sim 1 \mathrm{~mm}$ of the central retina area on the OCT map. Mean retinal sensitivity over the area with iFAF was determined by means of dedicated software (within the MP1) which allows for a semiautomatic overlapping of the microperimetric results with the FAF images (Figs. 2, 3).

\section{Statistical Methods}

Descriptive Statistics. Study parameters were described by means of usual methods of descriptive statistics: mean value, SD, minimum, maximum, and quantiles were tabulated for quantitative parameters; frequency and percentage were reported for qualitative ones.

Analytical Methods. An analysis of linear correlation was preliminarily conducted to measure the magnitude of relationships among variables by means of Pearson's correlation coefficient. Results of such analysis were taken into account while choosing parameters to include in the multiple analysis models.

The association of OCT edema patterns as well as FA edema patterns with iFAF was evaluated by means of Fisher's exact test applied to the distribution of frequency of each pattern by FAF group. Agreement between the same pattern as classified by OCT and FA was measured by means of the $\kappa$ statistics and $95 \%$ CI.

The significance of the relationship between parameters and FAF groups was evaluated by means of three-way analysis of covariance (ANCOVA). In the ANCOVA model FAF groups, OCT diffuse or focal edema pattern and cystoid or noncystoid edema pattern were included
FIGURE 1. Foveal FAF patterns in DME: normal FAF (left); single-spot iFAF (center); multiple-spot iFAF (right).
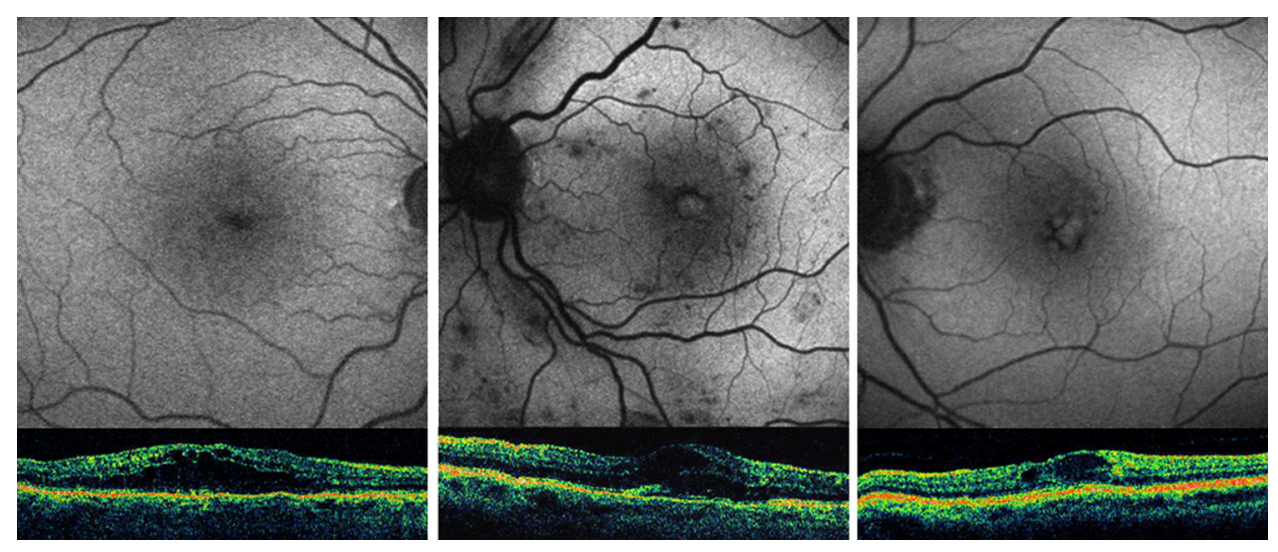

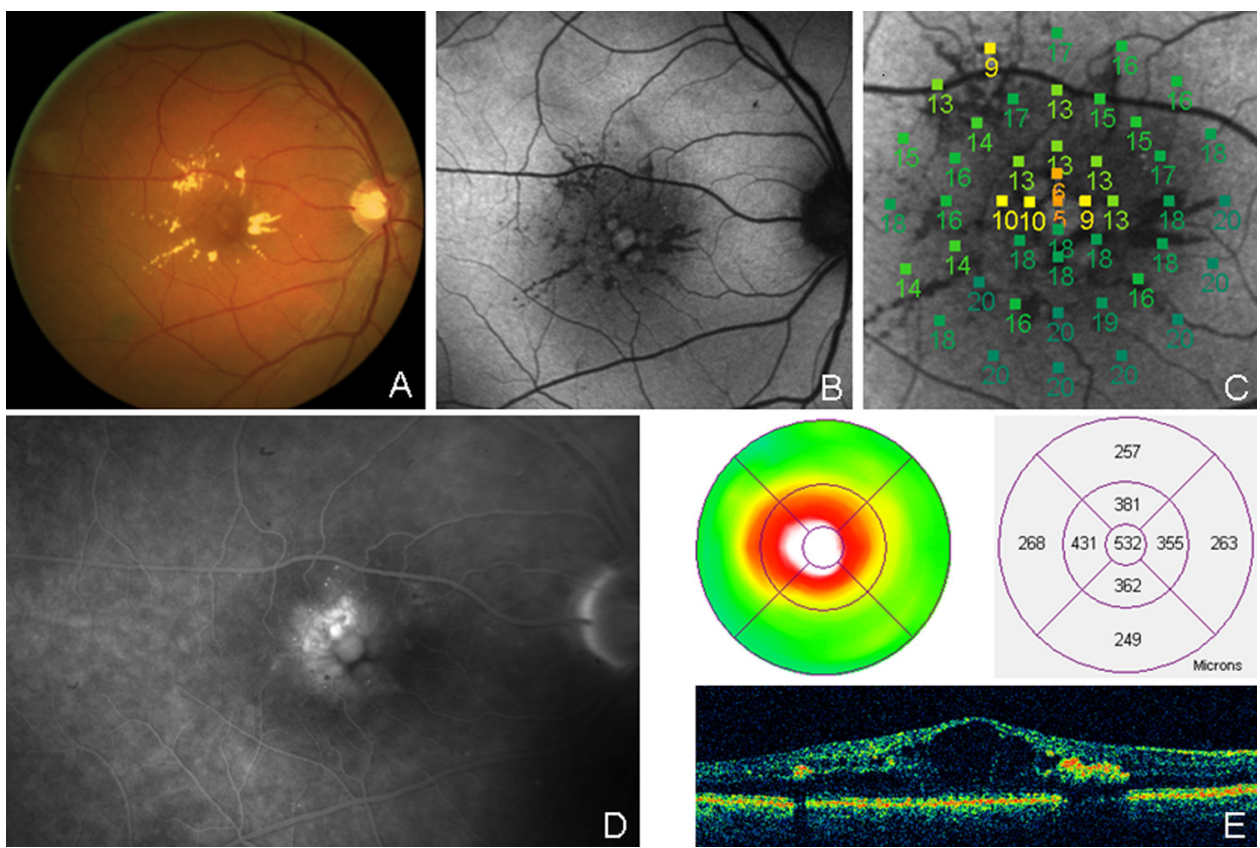

FigURE 2. Clinically significant DME with large foveal cysts (right eye): (A) Color fundus photo; (B) FAF showing a pattern of multiple-spot iFAF; (C) microperimetry sensitivity map overlaid on the FAF image; (D) fluorescein angiography confirming cystoid macular edema; and (E) OCT (retinal thickness map and line scan). Mean retinal sensitivity in the central $4^{\circ}(\sim 1 \mathrm{~mm})$ was $12.6 \mathrm{~dB}$; BCVA was $+0.24 \log$ Mar $(0.6$ Snellen equivalent). as main factors; BCVA, sensitivity over the area of iFAF, OCT macular thickness, and OCT macular volume were included as covariates. The Bonferroni post hoc test was used for further investigations regarding differences between mean values. Data were assumed significant if $P<$ 0.05 (SAS, ver. 9.1.3; SAS, Cary, NC).

\section{RESUlTS}

Of the 92 patients (151 eyes), 58 were men and 34 were women with a mean age of $63.0 \pm 11.0$ years (range, 31-83); 17 had type 1 diabetes mellitus (DM) and 75 had type 2 . Fifty-nine patients had both eyes eligible, whereas 33 patients had one eligible eye. Characteristics of all patients included in the study are shown in Table 1
Thirty-five (23.18\%) eyes had a normal FAF pattern, 48 (31.79\%) eyes single-spot iFAF, and 68 (45.03\%) eyes multiplespot iFAF. Intergrader agreement was almost perfect $(k=0.88$; $95 \% \mathrm{CI}, 0.81-0.93)$. Mean retinal sensitivity decreased over areas with iFAF and was $11.5 \pm 5.3 \mathrm{~dB}$ versus $15.1 \pm 3.9 \mathrm{~dB}$ with normal FAF (Table 1).

Of 151 eyes, 35 (23.2\%) had a focal edema pattern on FA and $116(76.8 \%)$, a diffuse pattern. A cystoid pattern was found in 105 (69.5\%) eyes on FA and in 102 (67.55\%) on OCT. A noncystoid (spongelike) pattern was found in 49 (32.5\%) eyes and subfoveal neuroretinal detachment in 24 (15.9\%) on OCT (Table 1).

All patients had central and stable fixation, as we have reported in DME patients. ${ }^{10}$ The mean duration of the MP1 examination was $9.1 \pm 2.3$ minutes
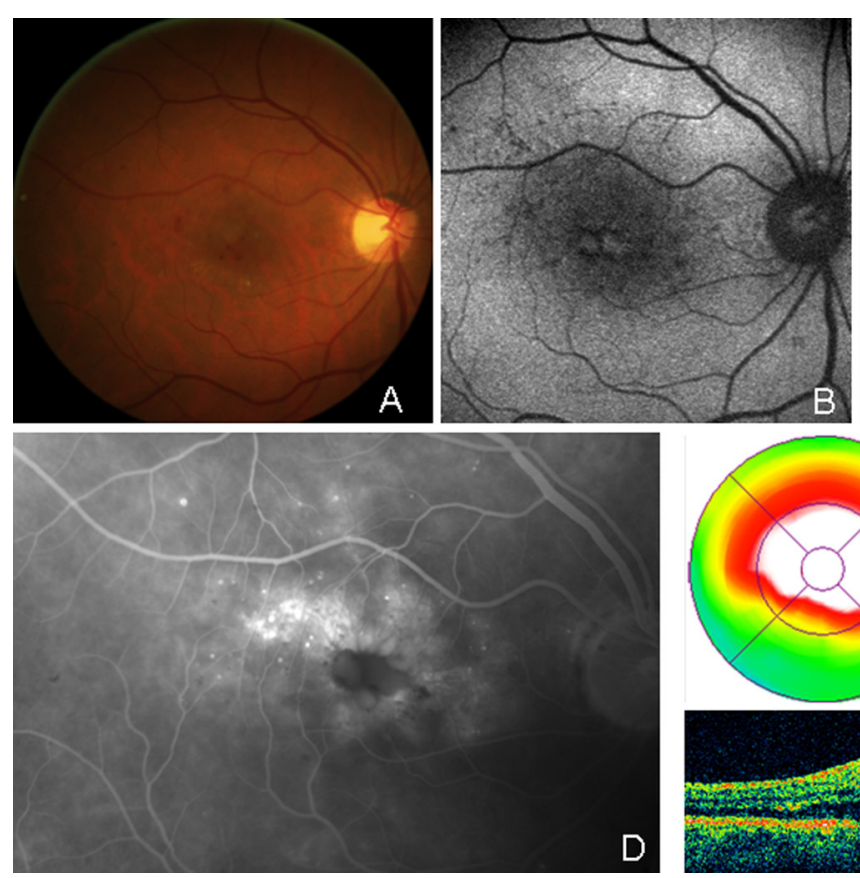
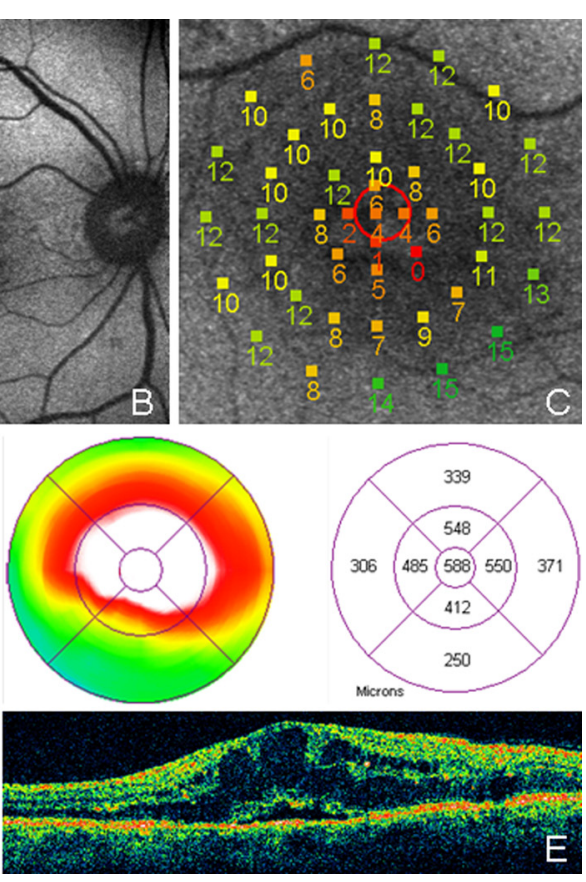

Figure 3. Clinically significant DME with large foveal cysts and subfoveal neuroretinal detachment (right eye): (A) Color fundus photo; (B) FAF showing pattern of multiple-spot iFAF; (C) microperimetry sensitivity map overlaid on the FAF image; (D) fluorescein angiography confirming cystoid macular edema; and (E) OCT (retinal thickness map and line scan). Mean retinal sensitivity in the central $4^{\circ}(\sim 1 \mathrm{~mm})$ was $5.5 \mathrm{~dB}$; BCVA was $+0.16 \log \operatorname{Mar}(0.7$ Snellen equivalent). 
TABLE 1. Characteristics of Patients with CSME

\begin{tabular}{|c|c|}
\hline \multicolumn{2}{|l|}{ Sex, $n(\%)$} \\
\hline Male & $58(63)$ \\
\hline Female & $34(37)$ \\
\hline \multicolumn{2}{|l|}{ Age, $y$} \\
\hline Range & $31-83$ \\
\hline Mean \pm SD & $63.0 \pm 11.0$ \\
\hline \multicolumn{2}{|l|}{ Type of DM, $n(\%)$} \\
\hline Type 1 & $17(15.2)$ \\
\hline Type 2 & $75(84.8)$ \\
\hline \multicolumn{2}{|l|}{ Duration of DM, $y$} \\
\hline Range & $1-50$ \\
\hline Mean \pm SD & $16.4 \pm 10.8$ \\
\hline HbA1c, \% & $8.0 \pm 1.8$ \\
\hline BCVA, logMAR & $0.227 \pm 0.284$ \\
\hline OCT central field thickness, $\mu \mathrm{m}$ & $332.9 \pm 106.3$ \\
\hline OCT volume, $\mathrm{mm}^{3}$ & $8.4 \pm 1.4$ \\
\hline \multicolumn{2}{|l|}{ FAF pattern, $n(\%)$} \\
\hline Normal & $35(23.2)$ \\
\hline Single spot & $48(31.8)$ \\
\hline Multiple spot & $68(45.0)$ \\
\hline Retinal sensitivity over areas without IFAF, dB & $15.1 \pm 3.9$ \\
\hline Retinal sensitivity over areas with iFAF, dB & $11.5 \pm 5.3$ \\
\hline \multicolumn{2}{|l|}{ FA edema patterns, $n(\%)$} \\
\hline Focal & $35(23.2)$ \\
\hline Diffuse & $116(76.8)$ \\
\hline Cystoid & $105(69.5)$ \\
\hline Noncystoid & $46(30.5)$ \\
\hline \multicolumn{2}{|l|}{ OCT edema patterns, $n(\%)$} \\
\hline Focal & $33(22.0)$ \\
\hline Diffuse* $^{*}$ & $117(78.0)$ \\
\hline Cystoid & $102(67.5)$ \\
\hline Spongelike & $49(32.5)$ \\
\hline Subfoveal neuroretinal detachment, $n$ & $24(15.9)$ \\
\hline
\end{tabular}

CSME, clinically significant macular edema.

* Information for one eye was missing.

\section{Correlation Analysis}

There was a strong, direct correlation between sensitivity over areas with iFAF and $4^{\circ}$ central sensitivity $(r=0.91)$. OCT volume showed strong, direct correlation with both OCT thickness $(r=0.68)$ and BCVA (logMAR; $r=0.42)$ and inverse correlation with sensitivity over iFAF areas $(r=-0.50)$. Lower correlation coefficients $(<0.40)$ were computed for the remaining relationships. Because of these correlations among parameters, in ANCOVA models, two parameters representing retinal function characteristics (BCVA and sensitivity over the area of iFAF) and two parameters representing retinal morpho- logic characteristics (OCT thickness and volume) were used as covariates.

\section{Association between FAF Pattern versus FA and OCT Edema Patterns}

Focal and diffuse FA patterns were associated with the FAF pattern (Fisher's exact test, $P=0.0072$ ). The OCT pattern was not significantly associated with the FAF pattern (Fisher's exact test, $P=0.7173$; Table 2 ).

Cystoid and noncystoid FA patterns were associated with FAF pattern (Fisher's exact test, $P<0.0001$ ) as well as OCT edema pattern (Fisher's exact test, $P<0.0001$; Table 2).

Agreement between OCT and FA in grading diffuse or focal and cystoid or noncystoid edema patterns was statistically significant: $\kappa=0.29(95 \% \mathrm{CI}, 0.14-0.44)$ and $\kappa=0.52(95 \%$ CI, $0.34-0.70)$, respectively.

\section{Association between FAF Pattern and Functional and Morphologic Parameters}

As shown by three-way ANCOVA, there was a significant difference in mean BCVA among three different FAF pattern groups (ANCOVA, $P=0.0494$ ), even if the Bonferroni post hoc test was unable to recognize specific differences between the mean values.

Mean retinal sensitivity over areas with iFAF was significantly different from that of normal FAF in both single- and multiple-spot iFAF groups (ANCOVA, $P=0.0002$; Bonferroni post hoc test, $P<0.01$ and $P<0.0001$, respectively). Mean retinal sensitivity progressively decreased in these three groups from $15.1 \pm 3.9$ to $10.3 \pm 5.2 \mathrm{~dB}(P=0.0002$; Table 3$)$. Mean $4^{\circ}$ central retinal sensitivity showed borderline statistical difference in all three FAF groups (ANCOVA, $P=0.0559$ ).

OCT CRT and volume were not significantly different between the FAF groups. Lesion area was not different between the iFAF groups (Table 3).

\section{Discussion}

This study documents that macular fundus autofluorescence increases in a large proportion (76.8\%) of patients with CSME. Moreover, retinal sensitivity decreases over areas with increased FAF, indicating that the function of the neurosensory retina deteriorates when autofluorescence increases.

DME is currently defined as increased retinal thickness. The functional impact of DME is usually determined by the quantification of BCVA. ${ }^{9,31}$ But, BCVA does not depend only on

TABLE 2. Description of OCT and FA Patterns by FAF

\begin{tabular}{|c|c|c|c|c|}
\hline & \multicolumn{3}{|c|}{ FAF Pattern } & \multirow[b]{2}{*}{$P$ (Fisher's Exact Test) } \\
\hline & Normal & Single-spot iFAF & Multiple-spot iFAF & \\
\hline \multicolumn{5}{|l|}{ OCT pattern } \\
\hline Focal & $9(27.3)$ & $11(33.3)$ & $13(39.4)$ & 0.7173 \\
\hline Diffuse & $26(22.2)$ & $36(30.8)$ & $55(47.0)$ & \\
\hline \multicolumn{5}{|l|}{ OCT pattern } \\
\hline Cystoid & $11(10.8)$ & $32(31.4)$ & $59(57.8)$ & $<0.0001$ \\
\hline Noncystoid & $24(49.0)$ & $16(32.6)$ & $9(18.4)$ & \\
\hline \multicolumn{5}{|l|}{ FA pattern } \\
\hline Focal & $20(57.7)$ & $7(20.0)$ & $8(22.3)$ & 0.0072 \\
\hline Diffuse & $26(22.4)$ & $35(30.2)$ & $55(47.4)$ & \\
\hline \multicolumn{5}{|l|}{ FA pattern } \\
\hline Cystoid & $14(13.3)$ & $33(31.4)$ & $58(55.3)$ & $<0.0001$ \\
\hline Noncystoid & $31(67.4)$ & $10(21.7)$ & $5(10.9)$ & \\
\hline
\end{tabular}

Data are $n(\%)$. 
TABLE 3. Description of Central Retina Characteristics by FAF Pattern

\begin{tabular}{lcccc}
\hline & & \multicolumn{2}{c}{ FAF Pattern } & \\
\cline { 3 - 4 } & $\begin{array}{c}\text { Normal } \\
(\boldsymbol{n}=\mathbf{3 5})\end{array}$ & $\begin{array}{c}\text { Single-spot } \\
\text { iFAF }(\boldsymbol{n}=\mathbf{4 8})\end{array}$ & $\begin{array}{c}\text { Multiple-spot } \\
\text { iFAF }(\boldsymbol{n}=\mathbf{6 8})\end{array}$ & $\begin{array}{c}\text { P (Three-way } \\
\text { ANOVA) }\end{array}$ \\
\hline BCVA, logMAR & $0.217 \pm 0.243$ & $0.175 \pm 0.213$ & $0.270 \pm 0.339$ & 0.0494 \\
Mean retinal sensitivity, dB & $15.1 \pm 3.9^{*} \dagger$ & $10.7 \pm 5.3^{*}$ & $10.3 \pm 5.2 \dagger$ & 0.0002 \\
$4^{\circ}$ CRS, dB & $15.1 \pm 3.9$ & $12.4 \pm 4.8$ & $11.4 \pm 4.9$ & 0.0559 \\
OCT thickness, $\mu \mathrm{m}$ & $295.9 \pm 100.5$ & $319.5 \pm 89.8$ & $361.5 \pm 113.4$ & 0.3680 \\
Volume, mm & $7.9 \pm 1.2$ & $8.4 \pm 1.4$ & $8.6 \pm 1.5$ & 0.8787 \\
Area of lesion, $\mathrm{mm}^{2}$ & $\mathrm{NA}$ & $0.172 \pm 0.199$ & $0.150 \pm 0.073$ & 0.1774 \\
\hline
\end{tabular}

Data are the mean \pm SD. CRS, central retina sensitivity; NA, not applicable.

${ }^{*} P<0.01 ; \dagger P<0.0001$ by Bonferroni post hoc test.

macular thickness. The Diabetic Retinopathy Clinical Research Network reported that for a given degree of diabetic maculopathy, a wide range of visual acuity may be observed. Therefore, changes in retinal thickness have low predictive value compared with BCVA changes.?

Microperimetry has recently been introduced in clinical practice for functional evaluation of DME. ${ }^{10-16}$ Microperimetry quantifies macular sensitivity, exactly correlating it to fundus characteristics, and determines retinal fixation characteristics. A significant inverse correlation between macular thickness and macular sensitivity has already been reported in many studies. ${ }^{12-15}$ More contrasting data exist about fixation characteristics in DME eyes ${ }^{10,11,14}$ - mostly due to the differences in examined populations, especially differences in DME duration. ${ }^{10,11,14}$ Vujosevic et al. ${ }^{10}$ reported in a well-defined group of CSME eyes that location and stability of fixation were normal, except when subfoveal hard exudates were present. Microperimetry may add some new information in the management of DME that is complementary to OCT and visual acuity data. ${ }^{12}$ Functional evaluation of DME is important for two reasons: first, morphologic evaluation is useful to assess the severity of DME and treatment outcomes, but it must correlate to visual function, to give better DME prognostication. Second, better correlation of structural and functional parameters may enable identification of new DME patterns, which may be responsible for different responses to local treatments. Therefore, treatments based on OCT macular thickness or FA leakage alone may be not as efficient as expected, giving rise to different outcomes. This fact may suggest the presence of different DME phenotypes. The determination of new patterns corresponding to these phenotypes may be useful in tailoring DME treatment.

Although classification of DME may vary when using different diagnostic methods and terms such as focal and diffuse DME are not yet standardized, several studies have recently shown strong correlations between OCT findings and FA patterns in DME. ${ }^{17,32-34}$ These correlations address the changes in intraretinal structure. Large foveal cysts, located in the outer nuclear layer and/or Henle's layer found on OCT correspond well to petaloid cystoid leakage patterns on FA. ${ }^{32,34}$ With the advent of new high-resolution OCT, fine intraretinal structure changes caused by cystic spaces have been visualized and could be better compared to histopathologic reports. ${ }^{35,36}$ In the present study, a high proportion of agreement in the evaluation of cystoid edema with OCT and FA was found, although a time-domain machine was used. On the contrary, OCT and FA disagree in detecting subfoveal neuroretinal detachment. Whereas SNRD is easily detected on OCT, it does not have any specific pattern on FA.

FAF has been used mostly for structural evaluation of agerelated macular degeneration, inherited macular dystrophies, and cystoid macular edema of different origins, but FAF char- acteristics in DME are still poorly understood. ${ }^{18-22,37-39}$ Bessho et al. ${ }^{37}$ documented increased FAF in all examined eyes with cystoid macular edema of different origin corresponding to the petaloid shape on FA and OCT cysts. Pece et al. ${ }^{38}$ described three different increased patterns of FAF (multicystic increased, single cyst increased, and combined single- and multicystic increased FAF) in patients with cystoid DME that correlated positively with FA and OCT findings. In the present study, FAF parameters correlated to both structural and functional parameters more commonly used in DME. The presence of iFAF was associated with functional and structural impairment of the macula. The iFAF pattern group had poorer macular sensitivity than did the normal FAF group. These data indicate that DME with iFAF pattern is, at least functionally, more severe than DME with a normal FAF pattern.

Other studies involved analysis of the correlation between FAF imaging and microperimetry in age-related macular degeneration, and the results are comparable to those obtained in this study. ${ }^{40,41}$ In particular, there was a significant correlation between presence of iFAF areas and decreased retinal sensitivity, regardless of visual acuity. ${ }^{40}$

In the present study, an increased FAF pattern (both singleand multiple-spot iFAF) was associated with cystoid edema pattern on OCT in approximately $89 \%$ of cases. Although the difference between single and multiple iFAF may sometimes be subtle, adequate clinical training overcomes this limitation. ${ }^{42}$ McBain et al. ${ }^{22}$ found high sensitivity and specificity of FAF examination in detecting cystoid edema, proposing FAF detection as a valid and noninvasive alternative to FA in evaluating cystoid macular edema of different origins.

The origin and significance of FAF in DME is still unclear. FAF is thought to visualize the distribution of lipofuscin in the RPE. ${ }^{20,43-45}$ In the latter, lipofuscin is mainly produced by incomplete degradation of photoreceptor outer segments, and it accumulates with age. ${ }^{43}$

In diabetic retinopathy, age-related accumulation of lipofuscin in the RPE, due to photoreceptor outer segment phagocytosis, does not appear to be a relevant pathogenic mechanism. But, lipofuscin contains a large number of molecules that are mainly peroxidation products of proteins and lipids. ${ }^{20}$ Thus, lipofuscin seems to be also an indicator of oxidative damage within the retina. A recent histologic study found that lipofuscin accumulates in microglia much more than in the RPE. ${ }^{46}$ This hypothesis could partly explain our results. It is wellknown that microglia are activated by diabetes; this activation could determine the oxidation of proteins and lipids that accumulate in the microglia due to ongoing diabetes. ${ }^{46}$ Thus, we hypothesize that the iFAF areas observed in DME are caused by accumulation of oxidative product induced by activated microglia. ${ }^{46}$

Another hypothesis is mainly related to the mechanical effect of cystoid macular edema: Cysts are mostly located in the 
outer plexiform and inner nuclear layers, where there is a maximum accumulation of luteal pigment. ${ }^{22}$ Cysts may displace luteal pigment preventing the normal blockage of foveal FAF signal at the level of each of them. ${ }^{22,45}$ Moreover, Bessho et al. ${ }^{37}$ documented increased FAF by 488 -nm excitation in all examined eyes with cystoid macular edema, but not by $580-\mathrm{nm}$ excitation, concluding that increased FAF is a pseudoautofluorescence due to a window defect, as in FA. As a consequence of this hypothesis, when cysts are reabsorbed, foveal FAF signal should disappear. This conclusion is in contrast with our experience in the follow-up of diabetic patients treated with laser photocoagulation in whom cysts reabsorbed but iFAF remained (Vujosevic S, et al. IOVS 2009;50:ARVO E-Abstract 1377). Although this phenomenon may be explained by residual displaced luteal pigment, it is also reasonable that it is related to persisting activated microglia.

However, more studies are needed to determine the exact origin of increased FAF signal in diabetes. FAF imaging could assist in identifying different DME patterns to improve macular edema classification and treatment in diabetic patients.

\section{Acknowledgments}

The authors thank Fabiano Cavarzeran, $\mathrm{ScD}$, for statistical analysis of the data.

\section{References}

1. Global prevalence of diabetes mellitus and its complications. In: Prevention of Blindness from Diabetes Mellitus. Geneva: WHO Report; 2005.

2. The Wisconsin Epidemiologic Study of Diabetic Retinopathy: XVII The 14-year incidence and progression of diabetic retinopathy and associated risk factors in type 1 diabetes. Ophthalmology. 1998; 105:1801-1815

3. Girach A, Lund-Andersen H. Diabetic macular oedema: a clinical overview. Int J Clin Pract. 2007;61:88-97.

4. Voutilainen-Kaunisto R, Teräsvirta M, Uusitupa M, Niskanen L. Maculopathy and visual acuity in newly diagnosed type 2 diabetic patients and non-diabetic subjects: a 10-year follow-up study. Acta Ophthalmol Scand. 2001;79:163-168.

5. Early Treatment Diabetic Retinopathy Study Research Group. Photocoagulation for diabetic macular edema. Early Treatment Diabetic Retinopathy Study report number 1. Arch Opthalmol. 1985; 103:1796-1806.

6. Strøm C, Sander B, Larsen N, Larsen M, Lund-Andersen H. Diabetic macular edema assessed with optical coherence tomography and stereo fundus photography. Invest Ophthalmol Vis Sci. 2002;43: 241-245.

7. Browning DJ, McOwen, Bowen RM, O'Marah TL. Comparison of clinical diagnosis of diabetic macular edema with diagnosis by optical coherence tomography. Ophthalmology. 2004;111:712-715.

8. Virgili G, Menchini F, Dimastrogiovanni AF, et al. Optical coherence tomography versus stereoscopic fundus photography or biomicroscopy for diagnosing diabetic macular edema: a systematic review. Invest Opbthalmol Vis Sci. 2007;48:4963- 4973.

9. Diabetic Retinopathy Clinical Research Network. Relationship between optical coherence tomography-measured central retinal thickness and visual acuity in diabetic macular edema. Ophthalmology. 2007;114:525-536.

10. Vujosevic S, Pilotto E, Bottega E, Benetti E, Cavarzeran F, Midena E. Retinal fixation impairment in diabetic macular edema. Reina. 2008; 10:1443-1450.

11. Carpineto P, Ciancaglini M, Di Antonio L, Gavalas C, Mastropasqua L. Fundus microperimetry patterns of fixation in type 2 diabetic patients with diffuse macular edema. Retina. 2007;27:21-29.

12. Vujosevic S, Midena E, Pilotto E, Radin PP, Chiesa L, Cavarzeran F. Diabetic macular edema: correlation between microperimetry and optical coherence tomography findings. Invest Ophthalmol Vis Sci. 2006;47:3044-3051.

13. Okada K, Yamamoto S, Mizunoya S, Hoshino A, Arai M, Takatsuna Y. Correlation of retinal sensitivity measured with fundus-related microperimetry to visual acuity and retinal thickness in eyes with diabetic macular edema. Eye. 2006;20:805-809.

14. Kube T, Schmidt S, Toonen F, Kirchhof B, Wolf S. Fixation stability and macular light sensitivity in patients with diabetic maculopathy: a microperimetric study with a scanning laser ophthalmoscope. Ophthalmologica. 2005;219:16-20.

15. Mori F, Ishiko S, Kitaya N, et al. Use of scanning laser ophthalmoscope microperimetry in clinically significant macular edema in type 2 diabetes mellitus. Jpn J Ophthalmol. 2002;46:650 - 655.

16. Rohrschneider K, Bültmann S, Glück R, Kruse FE, Fendrich T, Völcker HE. Scanning laser ophthalmoscope fundus perimetry before and after laser photocoagulation for clinically significant diabetic macular edema. Am J Ophthalmol. 2000;129: 27-32.

17. Browning DJ, Altaweel MM, Bressler NM, Bressler SB, Scott IU; Diabetic Retinopathy Clinical Research Network. Diabetic macular edema: what is focal and what is diffuse? Am J Ophthalmol. 2008; 146:649-655.

18. Bindewald A, Bird AC, Dandekar SS, et al. Classification of fundus autofluorescence patterns in early age-related macular disease. Invest Ophthalmol Vis Sci. 2005;46:3309-3314.

19. Vaclavik V, Vujosevic S, Dandekar SS, Bunce C, Peto T, Bird AC. Autofluorescence imaging in age-related macular degeneration complicated by choroidal neovascularization: a prospective study. Ophthalmology. 2008;115:342-346.

20. Schmitz-Valckenberg S, Holz FG, Bird AC, Spaide RF. Fundus autofluorescence imaging: review and perspectives. Retina. 2008; 28:385- 409 .

21. Spaide R. Autofluorescence from the outer retina and subretinal space: hypothesis and review. Retina. 2008;28:5-35.

22. McBain VA, Forrester JV, Lois N. Fundus autofluorescence in the diagnosis of cystoid macular oedema. Br J Ophthalmol. 2008;92 946-949.

23. Early Treatment Diabetic Retinopathy Study Research Group Grading diabetic retinopathy from stereoscopic color fundus photographs: an extension of the modified Airlie House classification: ETDRS report number 10. Ophthalmology. 1991;98:786-806.

24. Ferris FL, Kassoff A, Bresnick G, Bailey I. New visual acuity charts for clinical research. Am J Ophthalmol. 1982;94:91-96.

25. Bartsch DU, Weinreb RN, Zinser G, et al. Confocal scanning infrared laser ophthalmoscopy for indocyanine green angiography. Am J Ophthalmol. 1995;120:642-651.

26. Holz FG, Bellmann C, Margaritidis M, Schütt F, Otto TP, Völcker HE. Patterns of increased in vivo fundus autofluorescence in the junctional zone of geographic atrophy of the retinal pigment epithelium associated with age-related macular degeneration. Graefes Arch Clin Exp Ophthalmol. 1999;237:145-152.

27. Classification of diabetic retinopathy from fluorescein angiograms. ETDRS report number 11. Early Treatment Diabetic Retinopathy Study Research Group. Ophthalmology. 1991;98:807-822.

28. Otani T, Kishi S, Maruyama Y. Patterns of diabetic macular edema with optical coherence tomography. Am J Ophthalmol. 1999;127: 688-693.

29. Midena E, Radin PP, Pilotto E, Ghirlando A, Convento E, Varano M. Fixation pattern and macular sensitivity in eyes with subfoveal choroidal neovascularization secondary to age-related macular degeneration: a microperimetry study. Semin Ophthalmol. 2004; 19:55-61

30. Midena E, Radin PP, Convento E. Liquid crystal display microperimetry. In: Perimetry and the Fundus: an Introduction to Microperimetry. Midena E, ed. Thorofare, NJ: Slack, Inc.; 2007: 15-25.

31. Sakata K, Funatsu H, Harino S, Noma H, Hori S. Relationship of macular microcirculation and retinal thickness with visual acuity in diabetic macular edema. Ophthalmology. 2007;114:2061-2069.

32. Soliman W, Sander B, Hasler PW, Larsen M. Correlation between intraretinal changes in diabetic macular oedema seen in fluorescein angiography and optical coherence tomography. Acta Ophthalmol. 2008;86:34-39.

33. Neubauer AS, Chryssafis C, Priglinger SG, et al. Topography of diabetic macular oedema compared with fluorescein angiography. Acta Ophthalmol Scand. 2007;85:32-39.

34. Yeung L, Lima VC, Garcia P, Landa G, Rosen RB. Correlation between spectral domain optical coherence tomography findings 
and fluorescein angiography patterns in diabetic macular edema. Ophthalmology. 2009;116:1158-1167.

35. Fine BS, Brucker AJ. Macular edema and cystoid macular edema Am J Ophthalmol. 1981;92:466-481.

36. Yanoff M, Fine BS, Brucker AJ, Eagle RC Jr. Pathology of human cystoid macular edema. Surv Ophthalmol. 1984;28:505-511.

37. Bessho K, Gomi F, Harino S, et al. Macular autofluorescence in eyes with cystoid macula edema, detected with 488 nm-excitation but not with 580 nm-excitation. Graefes Arch Clin Exp Ophthalmol. 2009;247:729-734.

38. Pece A, Isola V, Holz F, Milani P, Brancato R. Autofluorescence imaging of cystoid macular edema in diabetic retinopathy. $O p h$ thalmologica. 2009;224:230-235.

39. Vujosevic S, Bottega E, Casciano M, Pilotto E, Convento E, Midena E. Microperimetry and fundus autofluorescence in diabetic macular edema: subthreshold micropulse diode laser versus modified early treatment diabetic retinopathy study laser photocoagulation. Retina. 2010;30:908-916.

40. Midena E, Vujosevic S, Convento E, Manfré A, Cavarzeran F, Pilotto E. Microperimetry and fundus autofluorescence in patients with early age-related macular degeneration. Br J Ophthalmol. 2007;91: $1499-1503$
41. Schmitz-Valckenberg S, Bültmann S, Dreyhaupt J, Bindewald A, Holz FG, Rohrschneider K. Fundus autofluorescence and fundus perimetry in the junctional zone of geographic atrophy in patients with age-related macular degeneration. Invest Ophthalmol Vis Sci. 2004; $45: 4470$ - 4476

42. Vujosevic S, Vaclavik V, Bird AC, Leung I, Dandekar S, Peto T. Combined grading for choroidal neovascularisation: colour, fluorescein angiography and autofluorescence images. Graefes Arch Clin Exp Ophthalmol. 2007;245:1453-1460.

43. Von Rückmann A, Fitzke FW, Bird AC. Distribution of fundus autofluorescence with a scanning laser ophthalmoscope. $\mathrm{Br} J$ Ophthalmol. 1995;79:407-412.

44. Delori FC, Dorey CK, Staurenghi G, Goger DG, Writer JJ. In vivo fluorescence of the ocular fundus exhibits retinal pigment epithelium lipofuscin characteristics. Invest Ophthalmol Vis Sci. 1995; 36:718-729.

45. Smith RT, Koniarek JP, Chan J, Nagasaki T, Sparrow JR, Langton K. Autofluorescence characteristics of normal foveas and reconstruction of foveal autofluorescence from limited data subsets. Invest Ophthalmol Vis Sci. 2005;46:2940-2946.

46. Xu H, Chen M, Manivannan A, Lois N, Forrester JV. Age-dependent accumulation of lipofuscin in perivascular and subretinal microglia in experimental mice. Aging Cell. 2008;7:58-68. 